\title{
Effect of Adding a Delayed Blastocyst to a Good Quality One during Embryo Transfer on ICSI Cycle Outcomes
}

\author{
Mohamed Elmahdy $^{1^{*}}\left(\mathbb{D}\right.$, Emadeldin Khalifa$^{1}$, Passant Radwan $^{2}$, Yasser Elkassar ${ }^{1}$ \\ ${ }^{1}$ Faculty of Medicine, Obstetrics and Gynecology Department, Alexandria University, Alexandria, Egypt \\ ${ }^{2}$ Egyptian Ministry of Health Hospitals, Alexandria, Egypt \\ Email: *mahdy_moh@yahoo.com
}

How to cite this paper: Elmahdy, M., Khalifa, E., Radwan, P. and Elkassar, Y. (2021) Effect of Adding a Delayed Blastocyst to a Good Quality One during Embryo Transfer on ICSI Cycle Outcomes. Open Journal of Obstetrics and Gynecology, 11, 1050-1063.

https://doi.org/10.4236/ojog.2021.118098

Received: July 2, 2021

Accepted: August 22, 2021

Published: August 25, 2021

Copyright $\odot 2021$ by author(s) and Scientific Research Publishing Inc. This work is licensed under the Creative Commons Attribution International License (CC BY 4.0).

http://creativecommons.org/licenses/by/4.0/

\begin{abstract}
Objectives: Study the effect of adding a delayed blastocyst to a transferred good quality one on ICSI cycle outcomes. Study design: Prospective cohort study. Participants/materials, setting, methods: 90 infertile patients aged from 20 - 35 years due to mild male factor, unexplained infertility or tubal factor. Patients with PCOS, endometriosis, RIF, poor responder and azoospermia were excluded. Setting: Duration 6 months from October 2019 to April 2020 in a private IVF center in Egypt. 30 cases were subjected to elective single embryo transfer and the other 60 with two embryo transfers, one good quality and another poor quality blastocyst. Results: (clinical pregnancy rate) was comparable between the two groups, being $47.7 \%$ and $36.7 \%$ in SET and DET groups respectively $(\mathrm{p}=0.361)$. Miscarriage and ectopic pregnancy rate were observed in $10 \%$ of the cases pregnant after SET (group I) and in only $6.7 \%$ of the cases pregnant after DET (group II) denoting no significant statistical difference between the two groups. The highest incidence was missed miscarriages in the two groups and ectopic pregnancy was observed only in one case in group II (DET). Twin pregnancy occurred in $22 \%$ of pregnant females in the second group. Late pregnancy complications in the form of preterm labour, premature rupture of membrane and preeclampsia occurred mainly in pregnant females in the second group $18 \%$. Conclusion: Neither adding a delayed blastocyst negatively affected the good one nor affected the live birth rate but increased multiple pregnancy rates.
\end{abstract}

\section{Keywords}

Embryo Transfer, ICSI, Delayed Blastocyst, Pregnancy Rate 


\section{Introduction}

IVF has become the most successful treatment of infertility. With continuous improvement of IVF protocols, implantation rates have greatly improved pushing us to shift our focus from implantation rates to properly counselling patients and implementing the practice of single embryo transfers in order to reduce the associated risks of multiple and high order pregnancies. Moreover, it is known from many publications that obstetric outcome after IVF is less favorable than natural conception [1].

Historically, multiple embryos were transferred to compensate for low rates of implantation. European and American registries of assisted reproduction consider a twin pregnancy rate of $>25 \%$ unacceptable and recommend reducing this incidence to $10 \%$ or lower [2]. Since the optimal outcome of an IVF cycle is the birth of a healthy singleton rather than a positive pregnancy test [3], thus implementing single embryo transfer (SET) is the only way to help us achieve this.

The definition of elective SET was stated by Gerris et al. in 2004 and in all randomized controlled trials (RCT) as transfer of one good quality embryo in cases where at least two or more good quality embryos were available and it was decided to intentionally transfer only one [4]. Transferring embryos one at a time would minimize the potential for all embryos to be transferred to a nonreceptive uterus as there exists variation in cycle-to-cycle endometrial receptivity in the form of endometrial thickness [5], progesterone levels [6] and histological configuration of the endometrium [7].

There is an everlasting concern of which is more important when applying SET strategy, decreasing overall implantation rates or reducing multiple pregnancies and their associated co-morbidities. The answer to this lies in considering the cumulative pregnancy rate which overcomes this decrease in implantation rate by an additional frozen single embryo transfer of the same stimulated cycle [8]. Thus the principle to balance this strategy and achieve optimal live birth rates is based on a strong blastocyst cryopreservation programme, selecting the best quality embryo for transfer and minimal prognostic criteria in selected patients.

The American Society of Reproductive Medicine (ASRM) assigned minimal good prognostic criteria to be available in SET candidates such as <35 years, more than one top-quality embryo available for transfer, excess embryos available for cryopreservation, outcome of any previous IVF cycles and availability of euploid embryos [9].

Moreover selecting the best embryo for transfer is an important factor influencing IVF outcome. In spite of its subjectivity, morphological evaluation is still the most widely used tool in embryo selection. Based on the ESHRE consensus, embryos at cleavage stage are classified as good, fair, and poor quality according to fragmentation rate, blastomere size and number, and the presence or not of multinucleated blastomeres [10].

A preposition to improving embryo selection is extending culture to blasto- 
cyst stage, but its grading is still far from standardized as blastocyst quality is still largely assessed based on appearance rather than viability. In spite of this Gardner classification until now remains largely unchallenged. It takes into account the expansion grade (score $1-6)$ and the development of the inner cell mass (ICM) and the trophectoderm (TE) each graded from (A-C) [11].

Outcome of blastocyst transfer is nearly $50 \%$ as this allows a natural selection and embryo assessment beyond genomic activation [12]. Blastocyst transfer provides better synchronization with uterine environment [13], spares exposure of early stage embryos to supra physiological concentrations of gonadotropins [14], allows time for reduction of uterine pulsatility post HCG injectiom to decrease expulsion [15] and greatly reduces the incidence of aneuploidies from 59\% to $35 \%[16]$.

Many studies have also proposed that when transferring two or more embryos along of different qualities, the poor ones may have a potential to impair implantation of the good ones through activating an oxidative stress response in endometrial stromal cells [17]. On the other hand others proved otherwise [18].

IVF coverage, country legislations, guideline recommendations, and patients education, counselling and culture are of utmost importance to apply SET.

\section{Objectives}

To compare ICSI outcome between cases transferring a single good quality embryo and cases transferring good and delayed embryo(s) on day 5 in the same cycle.

\section{Materials and Methods}

\subsection{Study Design}

This is a Prospective cohort study conducted in a private assisted reproductive centre in Alexandria, Egypt. The sample size included all patients attending the center from the period of 10/2019 to 5/2020 who met our inclusion criteria undergoing ICSI were included in the study after signing informed consent.

\subsection{Inclusion and Exclusion Criteria}

Female age limit was from 20 to 35 years. All patients were complaining of primary infertility and they were scheduled for intracytoplasmic sperm injection (ICSI). Any gynaecological pathology was treated before starting. Patients with Recurrent implantation failure, severe male factor, endometriosis, uterine cavity anomalies and polycystic ovaries (PCO) were excluded from the study.

\subsection{Treatment Protocols}

Flexible antagonist protocol was applied to all patients. All patients' follicular cohorts were synchronized using timed administration of an oral contraceptive (OC) containing $0.03 \mathrm{mg}$ of ethynil estradiol (EE) and $0.15 \mathrm{mg}$ of levonorgestrel (LNG). Then gonadotropins were administrated starting from day 2 cycle with a 
dose assigned according to patient's age, BMI, AMH and day 3 FSH. Gonadotropin dosage will be modified according to the follicular response. Approximately after the $6^{\text {th }}$ day of gonadotropin injection when follicular size reaches more than or equal to $14 \mathrm{~mm}$, subcutaneous administration of the $\mathrm{GnRH}$ antagonist (e.g., Cetrorelix) begins. Patient is routinely monitored via trans-vaginal sonography (TVS) and hormonal profiling of estrogen and progesterone levels of patients. When at least 3 follicles reach $>18 \mathrm{~mm}$, trigger and final maturation was induced using 10,000 units of HCG. After 35 hours of HCG injection the mature oocytes were retrieved under ultrasound guidance puncture with light general anasthesia.

\subsection{Oocyte Retrieval, Grading and Fertilization}

Retrieved oocytes were isolated from follicular fluid, denuded and classified into GV, MI and MII according to nuclear maturity. Each Oocyte of MII grade and of good quality was injected with a single immobilized sperm within 3 - 4 hours after retrieval based on semen quality. Embryo development was monitored once at $16-18 \mathrm{~h}$ for fertilization and pronuclear formation and at $28-30 \mathrm{~h}$ for early cleavage scoring. Embryos were incubated and monitored daily for blastomere number, size, symmetry and degree of fragmentation. The decision to culture to day 5 using sequential media was based on the quantity and quality of embryos on day 3 i.e. at least 8 cells. Day 3 embryos were excluded from the study and only harvested day 5 embryos were used.

\subsection{Blastocyst Grading}

On day 5 blastocyst quality and expansion were checked by 2 senior embryologists based on the Tripartite Scoring of Blastocyst proposed by Gardner and Schoolcraft. Grades from 1 - 6 were given to blastocysts based on the degree of blastocoel expansion and status of hatching. The numeric grading is followed by two Alphabetic grading (from AA to CC), for assessing the Inner Cell Mass (ICM) and Trophectoderm cells [11]. If necessary, assisted hatching with laser zona drilling $(20 \mathrm{~mm})$ was performed in embryos selected for embryo transfer. Patients who had only one blastocyst on day of transfer were also excluded as the single blastocyst transfer was nonelective.

\subsection{Embryo Transfer and Cryopreservation}

On the day of transfer, all patients were counselled properly on the quality and number of embryos they will transfer. After the decision, embryo transfer was done based on morphology using transcervical soft tip catheter under ultrasound guidance. Immediately after embryo transfer, catheters were flushed and the media inspected microscopically to confirm absence of embryos. Day 5 blastocysts were considered good quality while day 5 morula and cleavage embryos were considered poor. Cryopreservation was done for good quality supernumerary embryos with less than $30 \%$ cytoplasmic fragmentation. 


\subsection{Luteal Phase Support and Pregnancy Test Timing}

Luteal Support will be given in the form of $100 \mathrm{mg}$ IM progesterone in oil daily and $400 \mathrm{mg}$ progesterone pessaries. Serum Beta HCG will be tested after 14 days of transfer. In case of a positive pregnancy test, a transvaginal ultrasound will be performed 28 - 32 days after the embryo transfer and repeated as required. Clinical pregnancy will be confirmed if a fetal heartbeat was observed by transvaginal ultrasound.

\subsection{Outcomes}

Primary outcomes were clinical pregnancy rate which will be determined by observation of a gestational sac with fetal heart beat by transvaginal ultrasound at 6 weeks of pregnancy [18] and implantation rate which is defined as the number of gestational sacs observed at echographic screening at 6 weeks of pregnancy divided by the number of embryos transferred [19]. Secondary outcome were miscarriage and ectopic rate.

\section{Statistical Analysis}

Data were fed to the computer and analyzed using IBM SPSS software package version 20.0. (Armonk, NY: IBM Corp) Qualitative data were described using number and percent. Quantitative data were described using range (minimum and maximum), mean, standard deviation and median. Significance of the obtained results was judged at the 5\% level [20] [21].

The used tests were:

1) Chi-square test

For categorical variables, to compare between different groups.

2) Fisher's Exact or Monte Carlo correction

Correction for chi-square when more than $20 \%$ of the cells have expected count less than 5 .

3) Student t-test

For normally distributed quantitative variables, to compare between two studied groups.

4) Paired t-test

For normally distributed quantitative variables, to compare between two periods.

5) Mann Whitney test

For abnormally distributed quantitative variables, to compare between two studied groups.

6) Spearman coefficient

To correlate between two distributed abnormally quantitative variables.

Study cohorts: 90 patients will be included in the study. The study group will consist of 30 patients transferring single good quality embryo only [group 1]. The other group will include 60 patients transferring good and poor quality embryo(s) [group 2]. 


\section{Results}

Demographic data and cycle dynamic differences between the two groups were comparable between the two groups. The mean age for the whole study population was $31.13 \pm 3.76(\mathrm{p}=0.600)$. Most of the cases were overweight (BMI 25 29.9) and had their AMH > 1. The most common indication of ICSI in the two groups was the tubo-peritoneal factor which presented $26.7 \%$ in group I and $31.7 \%$ in group II. Thus, no significant statistical difference was found in any of these factors.

Comparing between the two groups regarding cycle characteristics as the number of oocytes retrieved, percentage of MII mature oocytes yielded in each group and fertilization rate in each was shown in Table 1. Thus, again none of these factors presented any significant statistical difference between the two groups.

Table 2 shows the comparison between the two studied groups regarding the quality of the transferred embryos. In both groups, the majority of good quality embryos were blastocysts grade 4AA. Thus, avoiding the quality of the good quality embryo being transferred to be a confounding factor between the two groups $(\mathrm{p}=0.089)$.

The implantation rate was significantly different between the two groups, being $100 \%$ in pregnant ladies after SET (group I) while it was only $50 \%$ in pregnant ladies after double embryo transfer (DET) (group II) ( $\mathrm{p} \leq 0.001$ ). Despite this difference the other outcome (clinical pregnancy rate) was comparable between the two groups, being $47.7 \%$ and $36.7 \%$ in SET and DET groups respectively $(\mathrm{p}=0.361)$, Table 3 .

Table 1. Comparison between the two studied groups according to oocytes retrieved, maturity and fertilization rate.

\begin{tabular}{ccccc}
\hline No. of oocytes & $\begin{array}{c}\text { SET } \\
(\mathrm{n}=30)\end{array}$ & $\begin{array}{c}\text { DET } \\
(\mathrm{n}=60)\end{array}$ & $\mathrm{U}$ & $\mathrm{p}$ \\
\hline Retrieved & 377 & 934 & & \\
Min. - Max. & $1.0-27.0$ & $6.0-40.0$ & & \\
Mean \pm SD. & $12.57 \pm 6.83$ & $15.57 \pm 7.61$ & 741.0 & 0.173 \\
Median & 13.50 & 14.0 & & \\
M2 oocytes & 275 & 618 & & \\
Min. - Max. & $1.0-23.0$ & $4.0-30.0$ & & \\
Mean \pm SD. & $9.17 \pm 5.45$ & $10.30 \pm 5.08$ & 803.50 & 0.407 \\
Median & 8.50 & 9.0 & & \\
Fertilization rate (\%) & & & & \\
Min. - Max. & $33.3-100.0$ & $20.0-100.0$ & & \\
Mean \pm SD. & $68.48 \pm 18.12$ & $72.2 \pm 18.04$ & 786.50 & 0.331 \\
Median & 71.0 & 72.05 & & \\
\hline
\end{tabular}

U: Mann Whitney test; p: p value for comparing between the studied groups. 
Table 2. Comparison between the two studied groups according to ET1.Q.

\begin{tabular}{ccccc}
\hline & \multicolumn{2}{c}{$\begin{array}{c}\text { SET } \\
(\mathbf{n}=30)\end{array}$} & \multicolumn{2}{c}{$\begin{array}{c}\text { DET } \\
(\mathbf{n}=60)\end{array}$} \\
\cline { 2 - 5 } ET1.Q & No. & $\%$ & No. & $\%$ \\
\hline 5AA & 1 & 3.3 & 2 & 3.3 \\
$4 \mathrm{AA}$ & 5 & 16.7 & 1 & 1.7 \\
$4 \mathrm{AB}$ & 14 & 46.7 & 21 & 35.0 \\
$4 \mathrm{BB}$ & 0 & 0.0 & 3 & 5.0 \\
$3 \mathrm{AA}$ & 0 & 0.0 & 2 & 3.3 \\
$3 \mathrm{AB}$ & 1 & 3.3 & 6 & 10.0 \\
$3 \mathrm{BB}$ & 1 & 3.3 & 0 & 0.0 \\
$2 \mathrm{AA}$ & 1 & 3.3 & 0 & 0.0 \\
$2 \mathrm{AB}$ & 1 & 3.3 & 5 & 8.3 \\
$2 \mathrm{BB}$ & 1 & 3.3 & 2 & 3.3 \\
$1 \mathrm{AA}$ & 0 & 0.0 & 2 & 3.3 \\
$\chi^{2}\left({ }^{\mathrm{MC}} \mathrm{p}\right)$ & 5 & 16.7 & 16 & 26.7 \\
\hline
\end{tabular}

$\chi^{2}$ : Chi square test; MC: Monte Carlo; $\mathrm{p}$ : $\mathrm{p}$ value for comparing between the studied groups.

Table 3. Comparison between the two studied groups according to clinical pregnancy and implantation rate (\%).

\begin{tabular}{|c|c|c|c|c|c|c|}
\hline & \multicolumn{2}{|c|}{$\begin{array}{c}\text { SET } \\
(n=30)\end{array}$} & \multicolumn{2}{|c|}{$\begin{array}{c}\text { DET } \\
(n=60)\end{array}$} & \multirow[t]{2}{*}{ Test of Sig. } & \multirow[t]{2}{*}{$\mathrm{p}$} \\
\hline & No. & $\%$ & No. & $\%$ & & \\
\hline \multicolumn{7}{|l|}{ Clinical pregnancy } \\
\hline Negative & 16 & 53.3 & 38 & 63.3 & \multirow{3}{*}{$\chi^{2}=0.833$} & \multirow{3}{*}{0.361} \\
\hline Positive & 14 & 46.7 & 22 & 36.7 & & \\
\hline Implantation rate (\%) & \multicolumn{2}{|c|}{$(\mathrm{n}=14)$} & \multicolumn{2}{|c|}{$(n=22)$} & & \\
\hline Min. - Max. & \multicolumn{2}{|c|}{$100.0-100.0$} & \multicolumn{2}{|c|}{$33.30-100.0$} & & \\
\hline Mean \pm SD & \multicolumn{2}{|c|}{$100.0 \pm 0.0$} & \multicolumn{2}{|c|}{$56.05 \pm 22.75$} & $\mathrm{U}=28.0^{*}$ & $<0.001^{*}$ \\
\hline Median & \multicolumn{2}{|c|}{100.0} & \multicolumn{2}{|c|}{50.0} & & \\
\hline
\end{tabular}

$\chi^{2}$ : Chi square test; U: Mann Whitney test; p: p value for comparing between the studied groups; ${ }^{*}$ : Statistically significant at $\mathrm{p} \leq 0.05$.

Other secondary outcomes were also observed in our study including missed miscarriage, miscarriage and ectopic pregnancy. It was presented in Table 4.

Rate of twinning in DET group (group II) only was 5 of 60 cases in DET group (group II) had multiple gestations (8.3\%). The 5 cases were dichorionic diamniotic. No cases of twins were registered in SET group (group I).

Complications to multiple gestation, that occurred only in group II (DET), in neonates ranged from preterm birth and low birth weight to NICU admission 
Table 4. Comparison between the two studied groups according to other outcomes.

\begin{tabular}{|c|c|c|c|c|c|c|}
\hline \multirow[t]{2}{*}{ Other outcomes } & \multicolumn{2}{|c|}{$\begin{array}{c}\text { SET } \\
(\mathrm{n}=30)\end{array}$} & \multicolumn{2}{|c|}{$\begin{array}{c}\text { DET } \\
(n=60)\end{array}$} & \multirow{2}{*}{$x$} & \multirow[t]{2}{*}{${ }^{\mathrm{FE}} \mathrm{p}$} \\
\hline & No. & $\%$ & No. & $\%$ & & \\
\hline No & 27 & 90.0 & 56 & 93.3 & \multirow{2}{*}{0.310} & \multirow{2}{*}{0.682} \\
\hline Yes & 3 & 10.0 & 4 & 6.7 & & \\
\hline Missed miscarriage & 2 & 6.7 & 3 & 5.0 & 0.106 & 1.000 \\
\hline Miscarriage & 1 & 3.3 & 0 & 0.0 & 2.022 & 0.333 \\
\hline Ectopic & 0 & 0.0 & 1 & 1.7 & 0.489 & 1.000 \\
\hline
\end{tabular}

$\chi^{2}$ : Chi square test; FE: Fisher Exact test.

and long-term developmental delay. While in mothers it ranged from premature preterm rupture of membranes to preeclampia and gestational diabetes mellitus. The highest incidence was incubation and NICU admission for preterms i.e., $<37$ weeks (5\%) and preeclampsia in the mothers 3.3\% (Table 5).

The relation between age and clinical pregnancy rate was comparable between the two groups with a $\mathrm{p}$ value of 0.899 denoting no significant statistical difference.

On the other hand, most cases with a positive clinical pregnancy rate were of AMH $>1$ but still no significant statistical difference was found here either ( $\mathrm{p}=$ 0.394) (Table 6).

\section{Discussion}

Demand for ART services increased placing pressure on IVF technologies to be more efficient. ICSI is a multistep invasive technique and its outcome is related to many factors raising an inevitable dilemma about which is the best approach in each step. One of the main issues during in vitro fertilization is the embryo quality yielded in every patient [22].

We carried out an extensive literature search and complied with existing guidelines. The overall quality of received data was good and there were few missing values. Our findings were adjusted for confounding factors such as maternal age, BMI, AMH and stage of the embryo which was all day 5 fresh blastocyst transfers. There is now a mounting body of evidence in favour of deferring the transfer of blastocysts and keeping them in culture until full expansion is achieved and transferring them in subsequent warming cycle [23].

With the great improvement in IVF outcomes comes the major arising complication of twins and higher order pregnancies with all their existing comobidities and mortalities. SET made it possible to decrease the multiple birth rates considerably, while keeping the overall pregnancy rate fairly constant.

Generally single embryo transfers are not a common practice in many developing countries due to many patient and physician factors. But the everlasting concern was, does implementing this strategy compromise pregnancy rates or 
Table 5. Distribution of the studied cases according to pregnancy course complications for multiple pregnancy in DET group $(n=60)$.

\begin{tabular}{ccc}
\hline Pregnancy course complications & No. & $\%$ \\
\hline PROM & 1 & 1.7 \\
PTLP & 2 & 3.3 \\
Incubation & 3 & 5.0 \\
PET & 2 & 3.3 \\
GDM & 0 & 0.0 \\
\hline
\end{tabular}

Table 6. Relation between clinical pregnancy and AMH in each group.

\begin{tabular}{|c|c|c|c|c|c|c|}
\hline \multirow{3}{*}{$\mathrm{AMH}$} & \multicolumn{4}{|c|}{ Clinical pregnancy } & \multirow{3}{*}{$x^{\square}$} & \multirow{3}{*}{$\mathrm{p}$} \\
\hline & \multicolumn{2}{|c|}{ Negative } & \multicolumn{2}{|c|}{ Positive } & & \\
\hline & No. & $\%$ & No. & $\%$ & & \\
\hline $\operatorname{SET}(\mathrm{n}=30)$ & \multicolumn{2}{|c|}{$(n=16)$} & \multicolumn{2}{|c|}{$(n=14)$} & \multirow{3}{*}{$6.467^{*}$} & \multirow{3}{*}{$0.011^{*}$} \\
\hline$<1$ & 12 & 75.0 & 4 & 28.6 & & \\
\hline$>1$ & 4 & 25.0 & 10 & 71.4 & & \\
\hline $\operatorname{DET}(n=60)$ & \multicolumn{2}{|c|}{$(n=38)$} & \multicolumn{2}{|c|}{$(n=22)$} & \multirow{3}{*}{$15.227^{\star}$} & \multirow{3}{*}{$<0.001^{*}$} \\
\hline$<1$ & 25 & 65.8 & 3 & 13.6 & & \\
\hline$>1$ & 13 & 34.2 & 19 & 86.4 & & \\
\hline $\mathrm{p}_{1}$ & \multicolumn{2}{|c|}{0.749} & \multicolumn{2}{|c|}{0.394} & & \\
\hline
\end{tabular}

$\chi^{2}$ : Chi square test; $\mathrm{p}: \mathrm{p}$ value for comparing between negative and positive in each group; ${ }^{*}$ Statistically significant at $\mathrm{p} \leq 0.05$.

not. Moreover, is it suitable with all patients and does embryo quality play a role in its success or not.

Our study was designed to question a common issue in the clinical practice of physicians dealing with IVF. What should we do with a poor-quality embryo? Is it right to transfer it along with a good quality embryo or will it impair its implantation potential?

Our cohort demonstrated that the live birth rates were similar either when a poor-quality embryo was transferred along with a good quality embryo or when a good quality embryo was transferred alone. Therefore, transferring a poor quality embryo with a good quality embryo did not decrease nor increases the chances for women to conceive, On the contrary it only increased the incidence of multiple gestations denoting that a poor quality embryo still has a chance for implantation [24]. This coincides with four recent studies by Wintner et al. in 2017 [18]. Dobson et al. in 2018 [25], Berkhout et al. in 2017 [26] and yanaihara et al. in 2008 [27].

Also a published RCT in 2006 on single versus double blastocyst transfer that included 48 patients randomized for one or the other regardless the embryo quality, showed no statistically significant difference in the pregnancy rates between the two groups [28]. Moreover, a systematic review and meta-analysis was 
done in 2010 yeilding similar results as our study in all collected trials. Gerris et al. [29], Martikainen et al. [30], Lukassen et al. [31] and Moustafa et al. [32] showed that the multiple pregnancy rates decreased significantly in the SET group (from $23 \%$ to $5 \%$ ) without a significant decline in the clinical pregnancy rates (from $39 \%$ to $34 \%$ ).

Our results contradict a recent study performed by El-danasouri et al. and Richardson et al., they found that transferring an impaired quality embryo along with a good quality embryo significantly lowered both the pregnancy and implantation rates than transferring the good quality embryo alone [18] [33].

Additionally, miscarriage rates were similar in the two groups. This is similar to other studies which have shown that once a clinical pregnancy was achieved with poor quality embryo, it had a similar chance of reaching live birth as a high-quality embryo [24].

Day 5 blastocyst transfers were used in this study as recent studies support embryo selection at the blastocyst stage as pregnancy rate of up to $50 \%$ can be obtained from it. In addition, a Cochrane Review demonstrated a significant difference in pregnancy rate in favour of blastocyst transfer in good prognostic patients in 9 of 18 randomized control trials reviewed [12].

The decision to perform DET rather than SET is not justified for patients that do not have two high-quality embryos available and any potential increase in pregnancy rate should be weighed against an increased multiple gestation rate in DET compared to SET.

In women under 35 years of age, transferring one fresh embryo and then, if needed, one frozen-and-thawed embryo dramatically reduces the rate of multiple births while achieving a rate of live births that is not substantially lower than the rate that is achievable with a double-embryo transfer [8]. In our study, we focused mainly on outcomes after a single fresh IVF cycle, cumulative outcomes of fresh followed by frozen single embryo transfers of the same stimulated cycle were not analysed in this study and is for future research.

According to our results, single embryo transfers (SET) should be the initial recommendation for selected patients with minimal prognostic criteria. Yet, when two embryos are transferred, women can be reassured that the quality of the second embryo does not seem to affect the pregnancy rate.

Lastly, Single embryo transfer challenges IVF providers to put the "art" back into ART. There is clearly an art to selecting appropriate patients, an art to selecting the best embryo for transfer and most importantly, there is the art of counselling patients. In order to move successfully toward single embryo transfer, we must truly master the art of the doctor-patient relationship.

Strength of the study: it was a prospective cohort study. Clinical pregnancy rate and implantation rates were calculated. All the study population was homogenous and we excluded confounding factors.

Limitations, reasons for caution: small sample size may affect the conclusions. Follow up for cumulative pregnancy rate in both groups is needed. Frozen cycles were not tracked to predict the cumulative outcome in our study. Ongoing 
pregnancy rate and live birth rates were difficult to track and recruit. Our study results were somewhat limited by the way the embryos were graded. Selecting the best embryo for transfer is still an ongoing challenge as it is still a subjective assessment, as morphological evaluation is still the most widely used tool in embryo selection. Perhaps more advanced methods to evaluate embryos, such as time lapse and genetic screening or pre-gestational screening (PGS) will provide better selection.

Wider implications of the findings: Encouragement of elective single blastocyst transfer policy for wider scale may improve cycle outcome and decrease risk of multiple pregnancy.

\section{Conclusion}

Elective SET is the most appropriate for those with a good prognosis: age $<35$ years, more than one top-quality embryo available for transfer, first or second treatment cycle or previous successful IVF cycles. DET with one delayed embryo plus one good quality embryo, at the blastocyst stage, does not increase the live birth rate but only increases multiple births. A delayed embryo does not negatively affect a good quality embryo, when transferred together in a double embryo transfer.

\section{Authors' Contributions}

ME, YE analyzed and interpreted the patient data regarding the quality of embryos at embryo transfer. EK, ME, YE and PR performed the ICSI procedure and the embryo transfer, and were contributors in writing the manuscript. All authors read and approved the final manuscript.

\section{Ethics Approval and Consent to Participate}

This study was approved from the Ethics Committee, Faculty of Medicine, Alexandria University. The number is: 0105646.

Written informed consent for publication of their clinical details and/or clinical images was obtained from the patient. A copy of the consent form is available for review by the Editor of this journal. The datasets used and/or analyzed during the current study are available from the corresponding author on reasonable request.

\section{Acknowledgements}

All IVF laboratory embryologists for assistance.

\section{Conflicts of Interest}

The authors declare no conflict of interest.

\section{References}

[1] Calhaz-Jorge, C., de Geyter, C., Kupka, M.S., de Mouzon, J., Erb, K., Mocanu, E., 
Motrenko, T., Scaravelli, G., Wyns, C. and Goossens, V. (2016) European IVF Monitoring Consortium (EIM) for the European Society of Human Reproduction and Embryology, 2012. Human Reproduction, 31, 1638-1652.

[2] ESHRE (2016) Assisted Reproductive Technology in Europe, 2012: Results Generated from European Registers by ESHRE.

[3] (2001) ESHRE Campus Course Report. Prevention of Twin Pregnancies after IVF/ICSI by Single Embryo Transfer. Human Reproduction, 16, 790-800.

[4] Gerris, J. (2004) Single Embryo Transfer and IVF/ICSI Outcome: A Balanced Appraisal. Human Reproduction Update, 11, 105-121.

[5] Richter, K.S., Bugge, K.R., Bromer, J.G. and Levy, M.J. (2007) Relationship between Endometrial Thickness and Embryo Implantation, Based on 1,294 Cycles of in $\mathrm{Vi}$ tro Fertilization with Transfer of Two Blastocyst-Stage Embryos. Fertility and Sterility, 87, 53-59. https://doi.org/10.1016/j.fertnstert.2006.05.064

[6] Bosch, E., Labarta, E., Crespo, J., Simon, C., Remohi, J., Jenkins, J., et al. (2010) Circulating Progesterone Levels and Ongoing Pregnancy Rates in Controlled Ovarian Stimulation Cycles for in Vitro Fertilization: Analysis of over 4000 Cycles. Human Reproduction, 25, 2092-2100. https://doi.org/10.1093/humrep/deq125

[7] Murray, M.J., Meyer, W.R., Zaino, R.J., Lessey, B.A., Novotny, D.B., Ireland, K., et al. (2004) A Critical Analysis of the Accuracy, Reproducibility, and Clinical Utility of Histologic Endometrial Dating in Fertile Women. Fertility and Sterility, 81, 1333-1343. https://doi.org/10.1016/j.fertnstert.2003.11.030

[8] Thurin, A., Hausken, J., Hillensjo, T., Jablonowska, B., Pinborg, A., Strandell, A., et al. (2004) Elective Single-Embryo Transfer versus Double-Embryo Transfer in in Vitro Fertilization. The New England Journal of Medicine, 351, 2392-2402. https://doi.org/10.1056/NEJMoa041032

[9] The Practice Committee of the American Society for Reproductive Medicine and the Practice Committee of the Society for Assisted Reproductive Technology (2017) Criteria for Number of Embryos to Transfer: A Committee Opinion. Fertility and Sterility, 99, 44-46.

[10] Van Royen, E., Mangelschots, K., De Neubourg, D., et al. (1999) Characterization of a Top Quality Embryo, a Step towards Single Embryo Transfer. Human Reproduction, 14, 2345-2349.

[11] Gardner, D.K. and Schoolcraft, W.B. (1999) In-Vitro Culture of Human Blastocysts. In: Jansen, R. and Mortimer, D., Eds., Towards Reproductive Certainty: Fertility and Genetics beyond 1999, The Parthenon Publishing Group, New York, 378-388.

[12] Blake, D.A., Farquhar, C.M., Johnson, N. and Proctor, M. (2007) Cleavage Stage versus Blastocyst Stage Embryo Transfer in Assisted Conception. Cochrane Database of Systematic Reviews, No. 6, CD002118.

[13] Martin, K.L. (2004) Blastocyst Culture-Clinical and Future Applications. Journal für Fertilität und Reproduktion, 14, 13-18.

[14] Glujovsky, D., Blake, D., Farquhar, C. and Bardach, A. (2012) Cleavage Stage versus Blastocyst Stage Embryo Transfer in Assisted Reproductive Technology. Cochrane Database of Systematic Reviews, 7, CD002118.

[15] Renato, F., Jean-Marc, A., Claudia, R., Franciois, O., Maria, S.L. and Rene, F. (2001) Uterine Contractility Decreases at the Time of Blastocyst Transfers. Human Reproduction, 16, 1115-1119. https://doi.org/10.1093/humrep/16.6.1115

[16] Catherine, S., Platteau, P., Van Assche, E., Michiels, A., Tournaye, H., Camus, M., et al. (2004) Comparison of Blastocyst Transfer with or without Preimplantation Ge- 
netic Diagnosis for Aneuploidy Screening in Couples with Advanced Maternal Age: A Prospective Randomized Controlled Trial. Human Reproduction, 19, 2849-2858. https://doi.org/10.1093/humrep/deh536

[17] El-Danasouri, I., Sterzik, K., Rinaldi, L., Pacchiarotti, A., DeSanto, M. and Selman, H. (2016) Effect of Transferring a Morphologically Impaired Embryo with a Good Quality Embryo on the Pregnancy and Implantation Rates. European Review for Medical and Pharmacological Sciences, 20, 394-398.

[18] Wintner, E.M., Hershko-Klement, A., Tzadikevitch, K., Ghetler, Y., Gonen, O., Wintner, O., et al. (2017) Does the Transfer of a Poor Quality Embryo Together with a Good Quality Embryo Affect the in Vitro Fertilization (IVF) Outcome? Journal of Ovarian Research, 10, 2.

[19] De Croo, I., Van der Elst, J., Everaert, K., De Sutter, P. and Dhont, M. (1998) Fertilization, Pregnancy and Embryo Implantation Rates after ICSI with Fresh or Frozen-Thawed Testicular Spermatozoa. Human Reproduction, 13, 1893-1897. https://doi.org/10.1093/humrep/13.7.1893

[20] Kotz, S., Balakrishnan, N., Read, C.B. and Vidakovic, B. (2006) Encyclopedia of Statistical Sciences. 2nd Edition, Wiley-Interscience, Hoboken.

[21] Kirkpatrick, L.A. and Feeney, B.C. (2013) A Simple Guide to IBM SPSS Statistics for Version 20.0. Student ed. Wadsworth, Cengage Learning, Belmont.

[22] Rosen, M.P., Shen, S., Shen, S., Rinaudo, P.F., Huddleston, H.G., McCulloch, C.E., et al. (2010) Fertilization Rate Is an Independent Predictor of Implantation Rate. Fertility and Sterility, 94, 1328-1333.

[23] Shapiro, B.S., Daneshmand, S.T., Desai, J., Garner, F.C., Aguirre, M. and Hudson, C. (2016) The Risk of Embryo-Endometrium Asynchrony Increases with Maternal Age after Ovarian Stimulation and IVF. Reproductive BioMedicine Online, 33, 50-55. https://doi.org/10.1016/j.rbmo.2016.04.008

[24] Oron, G., Son, W.Y., Buckett, W., Tulandi, T. and Holzer, H. (2014) The Association between Embryo Quality and Perinatal Outcome of Singletons Born after Single Embryo Transfers: A Pilot Study. Human Reproduction, 29, 1444-1451. https://doi.org/10.1093/humrep/deu079

[25] Dobson, S.J.A., Lao, M.T., Michael, E., Varghese, A.C. and Jayaprakasan, K. (2018) Effect of Transfer of a Poor Quality Embryo along with a Top Quality Embryo on the Outcome during Fresh and Frozen in Vitro Fertilization Cycles. Fertility and Sterility, 110, 655-660. https://doi.org/10.1016/j.fertnstert.2018.05.010

[26] Berkhout, R.P., Vergouw, C.G., van Wely, M., de Melker, A.A., Schats, R., Repping, S., et al. (2017) The Addition of a Low-Quality Embryo as Part of a Fresh Day 3 Double Embryo Transfer Does Not Improve Ongoing Pregnancy Rates. Human Reproduction Open, 2017, hox020.

[27] Yanaihara, A., Yorimitsu, T., Motoyama, H., Ohara, M. and Kawamura, T. (2008) Clinical Outcome of Frozen Blastocyst Transfer, Single vs. Double Transfer. Journal of Assisted Reproduction and Genetics, 25, 531-534.

[28] Fiddelers, A.A., van Montfoort, A.P., Dirksen, C.D., Dumoulin, J.C., Land, J.A., Dunselman, G.A., et al. (2006) Single versus Double Embryo Transfer: Cost Effectiveness Analysis alongside a Randomized Clinical Trial. Human Reproduction, 21, 2090-2097. https://doi.org/10.1093/humrep/del112

[29] Gerris, J., De Neubourg, D., Mangelschots, K., Van Royen, E., Van de Meerssche, M. and Valkenburg, M. (1999) Prevention of Twin Pregnancy after In-Vitro Fertilization or Intracytoplasmic Sperm Injection Based on Strict Embryo Criteria: A Prospective Randomized Clinical Trial. Human Reproduction, 14, 2581-2587. 
https://doi.org/10.1093/humrep/14.10.2581

[30] Martikainen, H., Tiitinen, A., Tomas, C., Tapanainen, J., Orava, M., Tuomivaara, L., et al. (2001) One versus Two Embryo Transfer after IVF and ICSI: A Randomized Study. Human Reproduction, 16, 1900-1903.

https://doi.org/10.1093/humrep/16.9.1900

[31] Lukassen, H.G., Braat, D.D., Wetzels, A.M., Zielhuis, G.A., Adang, E.M., Scheenjes, E., et al. (2005) Two Cycles with Single Embryo Transfer versus One Cycle with Double Embryo Transfer: A Randomized Controlled Trial. Human Reproduction, 20, 702-708. https://doi.org/10.1093/humrep/deh672

[32] Moustafa, M.K., Sheded, S.A. and El Aziz Mousta, M.A. (2008) Elective Single Embryo Transfer versus Double Embryo Transfer in Assisted Reproduction. Reproductive BioMedicine Online, 17, 82-87.

https://doi.org/10.1016/S1472-6483(10)60297-0

[33] Richardson, A., Moss, B., Humphrey, E., Davey, T., Zujovic, L., Hopkisson, J., et al. (2016) In Women Undergoing IVF/ICSI, Does the Addition of a Poor Quality Embryo Have a Detrimental Effect on Outcome during Double Embryo Transfer? $B J O G, 123,69$. 